\title{
ACCELERATION EFFICIENCY IN NONTHERMAL SOURCES \\ AND THE SOFT GAMMA-RAYS FROM NGC 4151 OBSERVED BY OSSE AND SIGMA
}

\author{
ANDRZEJ MACIOLEK-NIEDŹWIECKI and ANDRZEJ A. ZDZIARSKI \\ Copernicus Astronomical Center, Bartycka 18, 00-716 Warsaw, POLAND \\ and \\ ALAN P. LIGHTMAN \\ Dept. of Physics, MIT, Cambridge, MA 02139
}

\section{ABSTRACT}

We show that the recent observations of the Seyfert galaxy NGC 4151 in hard $\mathrm{X}$-rays and soft $\gamma$-rays by the OSSE and SIGMA detectors onboard CGRO and GRANAT, respectively, are well explained by a nonthermal model with acceleration of relativistic electrons at an efficiency of $\lesssim 50 \%$ and with the remaining power dissipated thermally in the source (the standard nonthermal $e^{ \pm}$pair model assumed $100 \%$ efficiency). Such an acceleration efficiency is generally expected on physical grounds. The resulting model unifies previously proposed purely thermal and purely nonthermal models. The pure nonthermal model for NGC 4151 appears to be ruled out. The pure thermal model gives a worse fit to the data than our hybrid nonthermal/thermal model. Our results are presented in Zdziarski, Lightman, \& Maciołek-Niedźwiecki (1993).

\section{References}

Zdziarski, A. A., Lightman, A. P., \& Maciołek-Niedźwiecki, A. 1993, ApJLetters, 414, L93

T. J.-L. Courvoisier and A. Blecha: Multi-Wavelength Continuum Emission of AGN, 379.

C 1994 IAU. Printed in the Netherlands. 